\title{
The role of the ocelli in the phototactic behaviour of the haematophagous bug Triatoma infestans
}

\author{
Claudio R. Lazzari *, Carolina E. Reiseman, Teresita C. Insausti \\ Departamento de Ciencias Biológicas, Facultad de Ciencias Exactas y Naturales, Universidad de Buenos Aires, Ciudad Universitaria, (1428) \\ Buenos Aires, Argentina
}

Received 24 February 1998; accepted 21 April 1998

\begin{abstract}
In addition to compound eyes, most adult insects posses two or three simple eyes, the ocelli. The function of these photoreceptors remains elusive in most cases. Triatomine bugs posses two well-developed ocelli, located in a latero-dorsal position, behind the compound eyes. We tested the role of the ocelli in the phototactic behaviour of Triatoma infestans, by measuring the time spent by adult males in the dark half of an experimental arena, which had the other half illuminated. The occlusion of the ocelli or the compound eyes alone had little effect on the phototactic response of the bugs. Only those insects which had both their ocelli and compound eyes occluded showed a significant reduction in their negative response to light. The ability of the ocelli of $T$. infestans to mediate the phototactic response by themselves (i.e., not through the modulation of compound eyes sensitivity) constitutes the first report on this function in insects. (C) 1998 Elsevier Science Ltd. All rights reserved.
\end{abstract}

Keywords: Triatoma infestans; Vision; Ocelli; Phototaxis; Behaviour

\section{Introduction}

The simple eyes of adult insects or ocelli have been the subject of numerous anatomical, physiological, and behavioural investigations. Different functional roles have been ascribed to these organs, in particular in those insects considered as "good flyers". They include modulation of the muscular tonus of the flight system, flight equilibrium control and the dorsal light response used during flight and also orientation in walking flies and ants (Goodman, 1970, 1981; Wehrhahn, 1984; Mizunami, 1994, 1995).

The two ocelli of Triatoma infestans (Klug) (Hemiptera: Reduviidae) are located in an unusual position, behind the compound eyes, looking dorsolaterally and frontally (Fig. 1A and 1B). The ocelli are well developed and bear prominent spherical lenses (about $455 \mu \mathrm{m}$ in diameter) overlying the photoreceptor layer (Insausti, 1997). The interneurones of the ocellar nerves continue inside the brain as a bilaterally symmetric tract,

* Corresponding author. Fax: + 54-1-782-0582/ + 54-1-544-7893; E-mail: lazzari@bg.fcen.uba.ar through the superficial dorsal protocerebrum. Their axons run between the calyces of the mushroom bodies and dorsal to the central body towards different regions of the brain, the suboesophageal and the thoracic ganglia. Descending first-order interneurones project to the contralateral posterior slope, deutocerebrum and suboesophageal, pro-, meso- and methathoracic centres (Insausti and Lazzari, 1996).

As mentioned, the functional role of the ocelli has been largely studied in good flying insects, such as bees, dragonflies and wasps. Although the ability of $T$. infestans to sustain quite long flights has been described (Lehane and Schofield, 1976; Ward and Baker, 1982), Triatominae are mainly walking insects that perform dispersing flights under certain conditions, e.g., starvation (Lehane and Schofield, 1982), and can hardly be ascribed as belonging to the "good flyers" group. This fact does not agree with the high degree of development observed in the ocellar system of T. infestans; a rhabdom, where the entire face of each retinula cell is modified to interdigitate each other giving place to a thick meshwork of rhabdomeres, a complex pigmentary system and direct connections to thoracic centres by firstorder interneurons are only some of their features (Insausti, 1997). 

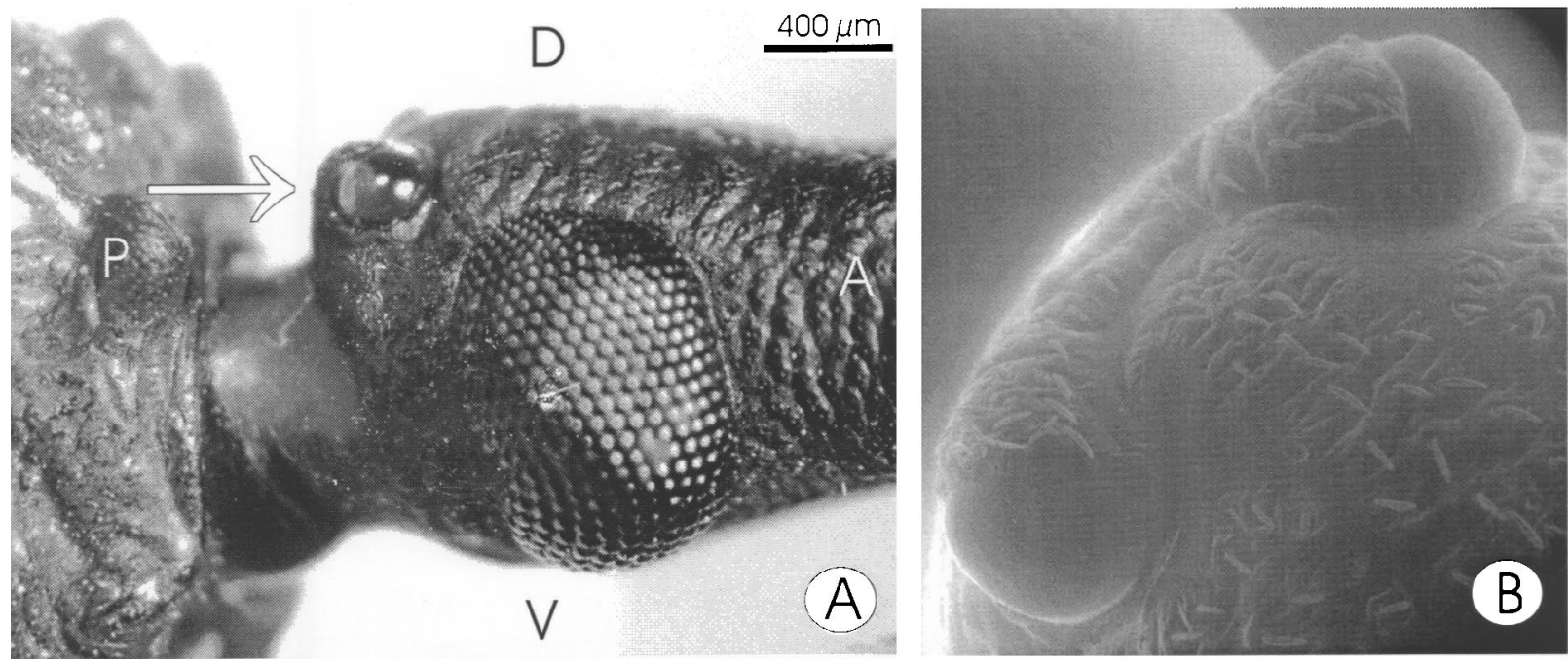

Fig. 1. The head and the visual organs of Triatoma infestans. (A), lateral view of the head; A, anterior; P, posterior; D, dorsal; V, ventral. The arrow points the right ocellus. (B) SEM photomicrograph of both ocelli.

One of the most typical responses of triatomines to light is their marked negative phototaxis (Ward and Finlayson, 1982; Reisenman and Lazzari, 1996). This behaviour seems to have a high adaptive value in an insect that spends daylight hours hidden in shelters, protected from near animals and humans that play the dual role of hosts and predators. $T$. infestans exhibits a high phototactic sensitivity, that varies under the control of a circadian endogenous clock (Reisenman and Lazzari, 1997; Reisenman et al., 1998).

Previous reports on different insect species indicate that the phototactic behaviour is mediated by visual inputs through the compound eyes, whereas the ocelli act as organs modulating the sensitivity of the former. In this work, we tested the role of the ocelli of $T$. infestans in the phototactic behaviour of this species.

\section{Material and methods}

Insects were reared in the laboratory at $28^{\circ} \mathrm{C}$ and fed weekly on heparinised bovine blood using an artificial feeder (Núñez and Lazzari, 1990). Adult males were used throughout.

Experiments were performed in a rectangular arena having filter paper as substrate and covered with a piece of rectangular glass (Fig. 2). Half of the arena was kept dark (0.04 lux) by means of fixing a piece of black cardboard to the glass cover; the other half remained illuminated (11.2 lux). Illumination was provided by a halogen, white light source (OSRAM 41860 WF, $12 \mathrm{~V} / 20 \mathrm{~W}$ ) placed inside an aluminium cylinder (diameter: $8 \mathrm{~cm}$, height: $17 \mathrm{~cm}$ ) that rested on top of a diffusing glass, $60 \mathrm{~cm}$ above the arena (Fig. 2). The aluminium cylinder avoided the spread of light out of the arena. The lamp was placed above the right or left end
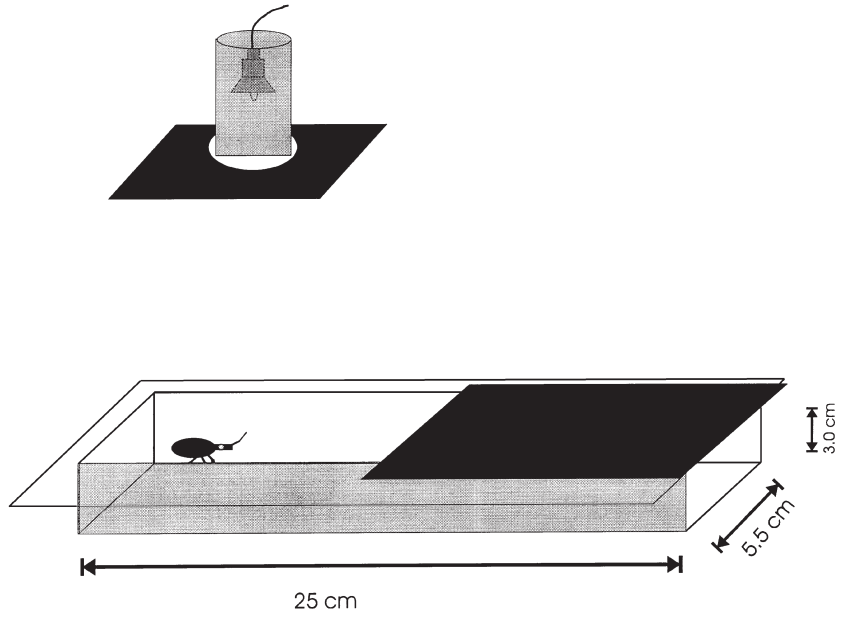

Fig. 2. Schema of the experimental arena used to test the phototactic response of Triatoma infestans. The floor of the arena was surfaced by filter paper as substrate and covered with a rectangular piece of glass. Half of the arena was kept dark (0.04 lux) by means of black cardboard fixed to the glass cover. The other half illuminated with 11.2 lux white light by means of an halogen lamp situated within an aluminium cylinder that rested on top of a diffusing glass, $60 \mathrm{~cm}$ above the arena. The aluminium cylinder allowed avoiding light spread in all possible directions but onto the arena.

of the arena in order to accentuate differences between its dark and illuminated halves and to avoid the establishment of a light gradient along it.

In each trial, one bug was released in the dark end of the arena at a time, the position of which (left or right) was changed every five trials, in order to avoid asymmetries. The insect was first placed within a small dark bowl that was, thereafter, carefully inverted on the arena. After a 40 seconds rest, the insect was gently released and the trial started.

Each trial lasted $5 \mathrm{~min}$; insects from the following groups were tested in an individual fashion: a, intact 
insects; $b$, insects having their ocelli occluded by a thick layer of black acrylic paint; c, insects having their compound eyes occluded and $d$, insects with both the ocelli and the compound eyes covered $(k=4, n=10)$.

\section{Results}

When released in the arena, all bugs displayed an exploratory behaviour, crossing the middle illumination boundary repeatedly. As expected, intact bugs spent most of the time of the trial in the dark half of the arena, whereas those having their visual inputs cancelled remained almost the same time in both halves. The occlusion of the ocelli or the compound eyes affected the phototactic behaviour of $T$. infestans by slightly reducing the permanent time in the shadow (Fig. 3).

Statistical analysis revealed a significant variation in the time spent in the dark half of the arena between groups (ANOVA test, $P<0.0002$ ). Multiple contrasts performed a posteriori indicated that the occlusion of the ocelli or the compound eyes alone had little effect on the phototactic response of the bugs (Tukey test, n.s.). Only those insects with both the ocelli and compound eyes occluded exhibited a significant reduction in their phototactic response which differed from all the other groups $(P<0.01)$, but not from a random distribution (i.e., $50 \%$ of the time spent in the dark side).

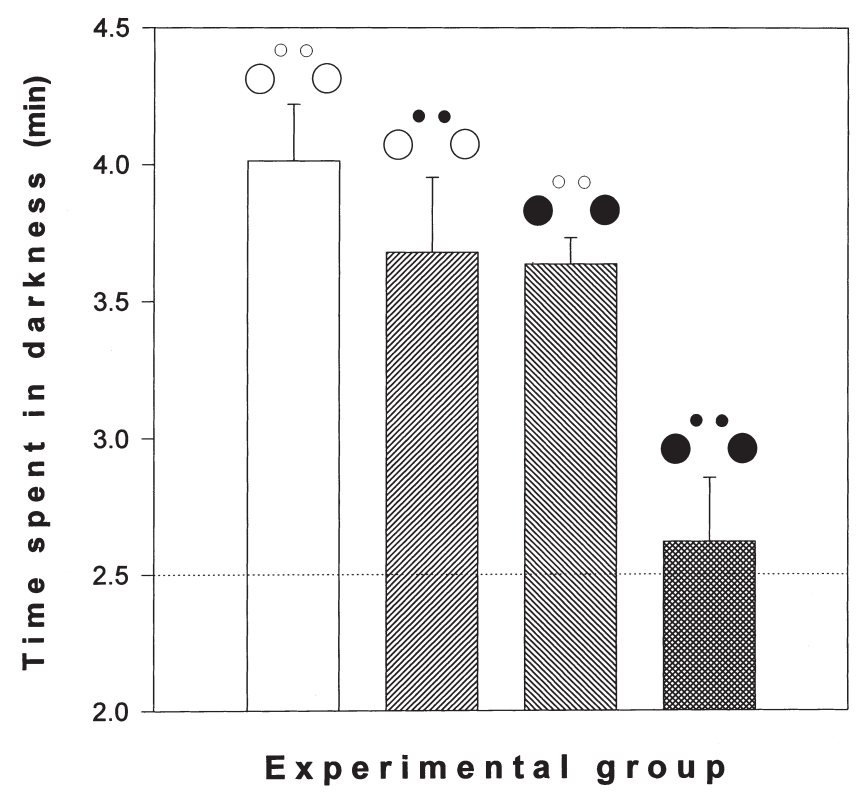

Fig. 3. Time spent in the dark half of the arena by intact adult males of $T$. infestans and by bugs having their ocelli and/or their compound eyes covered by a thick layer of black paint $(k=4, n=10)$. Mean and standard errors are depicted; maximum time $=5 \mathrm{~min}$. Significant differences between groups were evinced by the ANOVA test $(P<$ 0.0002). Multiple contrasts performed a posteriori revealed that only that group having both, ocelli and compound eyes statistically differed from the other groups (Tukey test, $P<0.01$ ), but not from a random distribution, (Tukey test, n.s.).

\section{Discussion}

Results reveal that ocelli can mediate the phototactic response by themselves in $T$. infestans, i.e., not through the modulation of compound eyes sensitivity (Fig. 3). Ocelli have been reported to modulate the sensitivity of compound eyes to light. However, the capacity to guide phototactic behaviour by themselves has rarely been mentioned (Mizunami, 1994) or even impossible (Goodman, 1970), based on studies performed in insects exhibiting positive phototaxis. So far as we know, this work is the first analysis of the ocellar function in relation to negative phototaxis and constitutes the first report on that capacity of insect ocelli.

This novel function of the ocelli of T. infestans is in agreement with the optical and structural properties of these photoreceptors. As in other insects, the focal plane of the ocellus of this species lies far below the retina, being unable to form an image. This characteristic, together with a meshwork-like rhabdom and the convergence of several thousands of photoreceptors in few firstorder interneurons, strongly suggests a high degree of spatial integration in these visual organs. In addition, the direct connection of the Triatoma ocelli with thoracic centres by first-order interneurons of large diameter are typical of a fast system (Insausti, 1997).

The above mentioned properties of the ocellar system become relevant in relation to the ability of bugs to detect shadows and illuminated areas and their directional reaction to them. The discovery of a shelter could depend, among others, on the light contrast between the illuminated environment and the darkness of the potential refuge, a task that would profit from high spatial integration. On the other hand, these bugs flee from a dark object moving in the panorama, a behaviour that requires a fast reaction capacity, i.e., fast connections between sensory and motor centres.

It is worth mentioning that two different orientation mechanisms are able to explain the longer time spent by the bugs in the dark side of the arena, with respect to the illuminated one. Insects could vary their walking speed according to the light intensity, walking slowly when in darkness and faster when in the illuminated side. This behaviour does not imply any response to the direction of the stimulus, but depends only on its intensity, i.e., photokinesis. The second mechanism involves a directional response to the stimulus source, i.e., phototaxis. Given that the light source was located above the animal, our experiments do not allow us to discern between both possibilities. Nevertheless, the behaviour of $T$. infestans under the conditions here employed has been analysed in detail in our laboratory previously (Reisenman and Lazzari, 1996; Reisenman et al., 1998). By measuring the locomotory activity of the bugs under different illumination conditions, it could be established that the 
walk speed does not vary, allowing photokinesis to be excluded.

Our findings stress the biological significance of the phototactic response in triatomines, which does not rely on a unique visual input. Other properties of this response, such as high sensitivity and dependence on an endogenous clock (Reisenman and Lazzari, 1997; Reisenman et al., 1998) also emphasise its adaptive value.

As seen in Fig. 3, both, ocelli and compound eyes are able to mediate by themselves the negative phototactic behaviour of $T$. infestans. The lack of one visual input has little effect on the response mediated by the other visual organs. This suggests the existence of parallel pathways each convening information from illumination of simple or compound eyes, that would converge in the same higher centre. Neuroanatomical studies have revealed direct connections between the ocellar neuropile and the lamina at the optic lobe of the protocerebrum by first-order interneurons in T. infestans (Insausti and Lazzari, 1996). These pathways provide a neural substrate for the ability of both, ocelli and compound eyes to mediate negative phototaxis in this species. The existence of parallel sensory pathways from different kinds of photoreceptors serving the same function adds a new element, that stresses the value of these insects as a model system for the study of the ocellar function.

\section{Acknowledgements}

Authors are indebted to G. Flores for correcting the manuscript and to the staff members of their Laboratory for many fruitful discussions. This work received support from the University of Buenos Aires, CONICET and FOSDIC (Argentina) and the WHO/UNDP/World Bank Special Programme for Research and Training in Tropical Diseases (TDR).

\section{References}

Goodman, L.J., 1970. The structure and function of the insect dorsal ocellus. Advances in Insect Physiology 7, 97-195.

Goodman, L.J., 1975. The neural organization and physiology of the insect dorsal ocellus. In: Horridge, G.A. (Ed.), The compound eye and vision of insects. Oxford University Press, Oxford.

Goodman, L.J., 1981. Organisation and physiology of the insect dorsal ocellar system. In: Autrum, H. (Ed.), Handbook of Sensory Physiology, vol VII/6C. Springer-Verlag, Berlin, Heidelberg and New York.

Insausti, T.C., 1997. Estudio del sistema ocelar de Triatoma infestans (Klug) 1834 (Heteroptera: Reduviidae) Doctoral Thesis, University of Buenos Aires. 259 pp.

Insausti, T.C., Lazzari, C.R., 1996. Central projections of first-order ocellar interneurons in Triatoma infestans (Heteroptera: Reduviidae). Journal of Morphology 229 (2), 161-169.

Lehane, M.J., Schofield, C.J., 1976. Preliminary report on flight by some triatomine bugs. Transactions of the Royal Society of Tropical Medicine and Hygiene 70, 526.

Lehane, M.J., Schofield, C.J., 1982. Flight initiation in Triatoma infestans (Klug) (Hemiptera: Reduviidae). Bulletin of Entomological Research 72, 497-510.

Mizunami, M., 1994. Information processing in the insect ocellar system: Comparative approaches to the evolution of visual processing and neural circuits. Advances in Insect Physiology 25, 151-265.

Mizunami, M., 1995. Functional diversity of neural organization in insect ocellar systems. Vision Research 4, 443-452.

Reisenman, C.E., Lazzari, C.R., 1996. The phototactic response of Triatoma infestans. Memórias do Instituto Oswaldo Cruz 91 (Suppl.), 156

Reisenman, C.E., Lazzari, C.R., 1997. A circadian rhythm of sensitivity in the eyes of Triatoma infestans as evinced by a behavioural assay. Memórias do Instituto Oswaldo Cruz 92 (Suppl.), 417.

Reisenman, C.E., Lazzari, C.R., Insausti, T.C., Giurfa, M., 1998. Attributes of the visual system of Triatoma infestans (Heteroptera) as revealed by the phototactic response. In: Wehner, R., Elsner, N., (eds.), New Neuroethology on the Move. Georg-Thieme-Verlag, Stuttgart-New York, p. 394

Ward, J.P., Baker, P.S., 1982. The tethered flight performance of a laboratory population of Triatoma infestans (Klug) (Hemiptera: Reduviidae). Bulletin of Entomological Research 72, 17-28.

Ward, J.P., Finlayson, L.H., 1982. Behavioural responses of the haematophagous bug Triatoma infestans (Klug) (Hemiptera: Reduviidae) to visual stimuli. Bulletin of Entomological Research 72, 357-366

Wehrhahn, C., 1984. Ocellar vision and orientation in flies. Proceedings of the Royal Society of London B 222, 409-411. 